\title{
SemIndex: Semantic-Aware Inverted Index
}

\author{
Richard Chbeir ${ }^{\star 1}$, Yi Luo ${ }^{2}$, Joe Tekli ${ }^{3}$, Kokou Yetongnon ${ }^{2}$, Carlos Raymundo \\ Ibañez $^{4}$, Agma J. M. Traina ${ }^{5}$, Caetano Traina Jr. ${ }^{5}$, and Marc Al Assad ${ }^{3}$ \\ 1 University of Pau and Adour Countries, Anglet, France \\ ${ }_{2}^{2}$ University of Bourgogne, Dijon, France \\ 3 Lebanese American University, Byblos, Lebanon \\ 4 Universidad Peruana de Ciencias Aplicadas, Lima, Peru \\ ${ }^{5}$ University of São Paulo, São Carlos-SP, Brazil
}

\begin{abstract}
This paper focuses on the important problem of semanticaware search in textual (structured, semi-structured, NoSQL) databases. This problem has emerged as a required extension of the standard containment keyword based query to meet user needs in textual databases and IR applications. We provide here a new approach, called SemIndex, that extends the standard inverted index by constructing a tight coupling inverted index graph that combines two main resources: a general purpose semantic network, and a standard inverted index on a collection of textual data. We also provide an extended query model and related processing algorithms with the help of SemIndex. To investigate its effectiveness, we set up experiments to test the performance of SemIndex. Preliminary results have demonstrated the effectiveness, scalability and optimality of our approach.
\end{abstract}

Keywords: Semantic Queries, Inverted lndex, NoSQL indexing, Semantic Network, Ontologies.

\section{Introduction}

Processing keyword-based queries is a fundamental problem in the domain of Information Retrieval (IR). Several studies have been done in the literature to provide effective IR techniques $[10,9,6]$. A standard containment keywordbased query, which retrieves textual identities that contain a set of keywords, is generally supported by a full-text index. Inverted index is considered as one of the most useful full-text indexing techniques for very large textual collections [10], supported by many relational DBMSs, and recently extended toward semistructured [9] and unstructured data [6] to support keyword-based queries.

Besides the standard containment keyword-based query, semantic-aware or knowledge-aware (keyword) query has emerged as a natural extension encouraged by real user demand. In semantic-aware queries, some knowledge ${ }^{6}$ needs to be taken into consideration while processing. Let's assume having a movie database, as shown in Table 1. Each movie, identified with an $i d$, is described with some (semi-structured) text, including movie title, year and plot. For queries "sound of music", "Maria nun" and "sound Maria", the query result is movie $\mathrm{O}_{3}$. However, if the user wants to search for a movie but cannot recall the exact movie title, it is natural to assume that (s)he may modify the query terms to some semantically similar terms, for example, "voice of music". Also, it is common that the terms provided by users are not exactly the same, but are semantically relevant to terms that the plot providers use. Clearly, the standard inverted index which only supports exact matching cannot deal with these cases. Various approaches combining different types of data and se-

\footnotetext{
* Corresponding author: rchbeir@acm.org/richard.chbeir@univ-pau.fr

${ }^{6}$ Also called domain, collaborative, collective knowledge or semantic network
} 
Table 1. A Sample Movie Data Collection

\begin{tabular}{|l|l|}
\hline ID & Textual Contents \\
\hline \hline$O_{1}$ & $\begin{array}{l}\text { When a Stranger Calls (2006): A young high school student babysits for a very rich family. She } \\
\text { begins to receive strange phone calls threatening the children... }\end{array}$ \\
\hline$O_{2}$ & $\begin{array}{l}\text { Code } R \text { (1977): This CBS adventure series managed to combine elements of "Adam-12", "Emer- } \\
\text { gency" and "Baywatch" at the same time... }\end{array}$ \\
\hline$O_{3}$ & $\begin{array}{l}\text { Sound of Music, The (1965): Maria had longed to be a nun since she was a young girl, yet when } \\
\text { she became old enough discovered that it wasn't at all what she thought... }\end{array}$ \\
\hline$\cdots$ & .. \\
\hline
\end{tabular}

mantic knowledge have been propose to enhance query processing (cf. Related Works). In this paper, we present a new approach integrating knowledge into a semantic-aware inverted index called SemIndex to support semantic-aware querying. Major differences between our work and existing methods include:

- Pre-processing the index: Existing works use semantic knowledge to pre-process queries, such as query rewriting/relaxation and query suggestion $[2,5]$, or to post-process the query results, such as semantic result clustering $[16,17,25]$. Our work can be seen as another alternative to consider the semantic gap by enclosing semantic knowledge directly into an inverted index, so that main tasks can be done before query processing,

- User involvement: Most existing works introduce some predefined parameters (heuristics) to rewrite queries such that users are only involved in the query refinement (expansion, filtering, etc.) process after providing the first round of results $[3,4,14,20]$. In our work, we aim at allowing end-users to write, using the same framework, classical queries but also semantically enriched queries according to their needs. They are involved in the whole process (during initial query writing and then query rewriting).

- Providing more results: Most existing works focus on understanding the meaning of dat/queries through semantic disambiguation $[2,13,16]$, which: i) is usually a complex process requiring substantial processing time [15], and ii) depends on the query/data context which is not always sufficiently available, and thus does not guarantee correct results $[7,23]$. The goal of our work is, with the help of semantic knowledge, to find more semantically relevant results than what a traditional inverted index could provide, while doing it more efficiently than existing disambiguation techniques.

In order to build SemIndex, we create connections between two data resources, $a$ textual data collection and a semantic knowledge base, and map them into a single data structure. An extended query model with different levels of semantic awareness is defined, so that both semantic-aware queries and standard containment queries are processed within the same framework. Figure 1 depicts the overall framework of our approach and its main components. The Indexer manages SemIndex, while the Query Processor accepts semantic-aware queries from the user and processes the queries with SemIndex.

The rest of this paper is organized as follows. Designing and building $S e$ $m$ Index will be presented in Section 2 . Section 3 will introduce our query model for semantic-aware queries. We will also present the algorithms for processing semantic-aware queries using SemIndex. We provide preliminary experimental results of executing different queries on a set of textual data collections in Section 4. Related works are discussed in Section 5 before concluding the paper and providing some future works. 


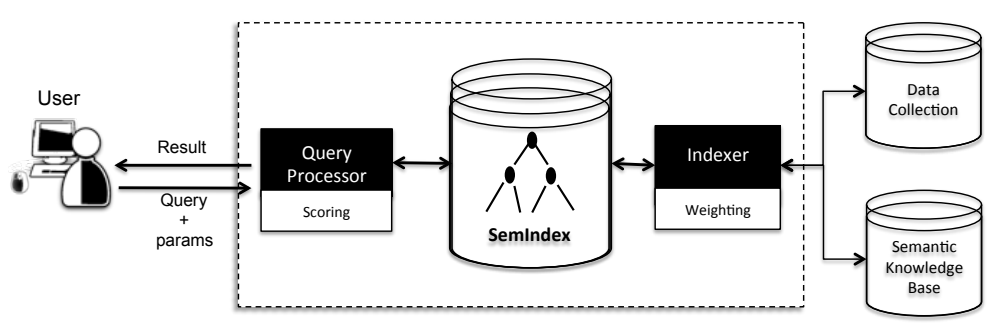

Fig. 1. SemIndex Framework

\section{Index Design}

In the following, we analyze the (textual and semantic) input resources required to build our index structure, titled SemIndex. We also show how to create connections between the input resources and how to design the logical structure of SemIndex. The physical structure will be detailed in a dedicated paper.

\subsection{Representation and Definitions}

Textual Data Collection: In our study, a textual data collection could be a set of documents, XML fragments, or tuples in a relational or NoSQL database.

Definition $1 A$ Textual Data Collection $\Delta$ is represented as a table defined over a set of attributes $\mathcal{A}=\left\{A_{1}, \ldots, A_{p}\right\}$ where each $A_{i}$ is associated with a set of values (such as strings, numbers, BLOB, etc.) called the domain of $A_{i}$ and denoted by $\operatorname{dom}\left(A_{i}\right)$. A semantic knowledge base $(K B)$ can be associated to one or several attribute domains $\operatorname{dom}\left(A_{i}\right)$. Thus, given a table $\Delta$ defined over $\mathcal{A}$, objects $t$ in $\Delta$ are denoted as $\left\langle a_{1}, \ldots, a_{p}\right\rangle$, where $a_{i} \in \operatorname{dom}\left(A_{i}\right)$. Each $a_{i}$ from $t$ is denoted as t. $a_{i}$.

Semantic Knowledge-Base: We adopt graph structures for modeling semantic knowledge. Thus, entities are represented as vertices, and semantic relationship between entities are modeled as directed edges ${ }^{7}$. In this work, we will illustrate the design process of SemIndex using WordNet version $3.0[8]$ as the semantic knowledge resource. Part of the WordNet ontology is shown in Figure 2. Each synset represents a distinct concept, and is linked to other synsets with semantic relations (including hypernymy, hyponymy, holonymy, etc.). Note that multiple edges may exist between each ordered pair of vertices, and thus the knowledge graph is a multi-graph.

Definition $2 A$ Semantic Knowledge Base $K B$, such as WordNet, is a graph $G_{k b}\left(V_{k b}, S_{k b}, E_{k b}, L_{k b}\right)$ such that:

- $V_{k b}$ is a set of vertices/nodes, denoting entities in the given knowledge base. For WordNet, $V_{k b}$ includes synsets and words

- $S_{k b}$ is a function defined on $V_{k b}$, representing the string value of each entity

- $E_{k b}$ is a set of directed edges; each has a label in $L_{k b}$ and is between a pair of vertices in $V_{k b}$

- $L_{k b}$ is a set of edge labels. For WordNet, $L_{k b}$ includes hyponymy, meronymy, hypernymy, holonymy, has-sense and has-sense-inverse, etc.

\footnotetext{
$\overline{7}$ We use the terms "edge" and "directed edge" interchangeably in this paper
} 
In Figure 2, $W_{4}, W_{6}, W_{7}, W_{8}$ and $W_{9}$ represent words, and their string values (lemma of the words) are shown aside of the nodes. $S_{1}, S_{3}$ and $S_{4}$ are synsets, and their string values are their definitions. If one sense of a word belongs to a synset, it is represented with two edges between the synset node and word node with opposite directions, labeled has-sense and has-word, that we represent here with only one left-right arrow.

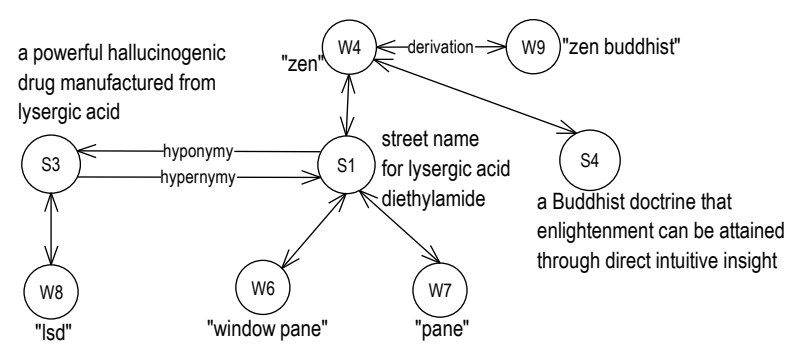

Fig. 2. Part of the Semantic Knowledge Graph of WordNet

SemIndex graph: To combine our resources, we define a SemIndex graph.

Definition 3 A SemIndex graph $\tilde{G}$ is a directed graph $\left(V_{i}, V_{d}, L, E, S_{v}, S_{e}, W\right)$ :

- $V_{i}$ is a set of index nodes (denoting entities in a knowledge-base, index or searchable terms in a textual collection) represented visually as circles $\bigcirc$

- $V_{d}$ is the set of data nodes (belonging to the textual collection) represented visually as squares

- $L$ is a set of labels

- $E$ is a set of ordered pairs of vertices in $V_{i} \cup V_{d}$ called edges. Edges between index nodes are called index edges (represented visually as $\rightarrow$ ), while edges between index nodes and data nodes are called data edges (represented visually as $\rightarrow \rightarrow$ )

- $S_{v}$ is a function defined on $V_{i} \cup V_{d}$, representing the value of each node

- $S_{e}$ is a function defined on $E$, assigning a label $\in L$ to an edge

- $W$ is a weighting function defined on nodes in $V_{i} \bigcup V_{d}$ and edges in $E$.

\subsection{Logical Design}

In this part, we introduce the logical design techniques in building SemIndex.

Building SemIndex: SemIndex adapts tight coupling techniques to index a textual data collection and a semantic knowledge base in the same framework, and directly create a posting list for all searchable contents. In the following, we describe how to construct SemIndex.

1- Indexing Input Resources: Given a textual data collection $\Delta$, a multiattributed inverted index (associated to one or several attributes $A$ ) is a mapping $i i: V_{A}^{s} \rightarrow V^{t}$, where $V_{A}^{s}$ is a set of values for attributes $A$, which we also call a searchable terms, and $V^{t}$ is the set of textual data objects. A multiattributed inverted index of a set of textual data objects $\Delta$ is represented as a SemIndex graph $\tilde{G}_{A}$ such that: 
- $V_{i}$ is a set of index nodes, representing all searchable terms which appear in the attribute set $A$ of the collection

- $V_{d}$ is the set of textual data objects in $\Delta$

- $L$ includes contained label indicating the containment relationship from a searchable term in $V_{i}$ to a data object in $V_{d}$

- $E$ is a set of ordered pairs of vertices in $V_{i} \bigcup V_{d}$

- $S_{v}$ assigns a term to an index node, and its text contents to a data node

- $S_{e}$ assigns the contained label to each edge

- $W$ assigns a weight (according to the importance/frequency of the term within the text content) to an edge $E$.

An example of an SemIndex graph inverted index $\tilde{G}_{A}$ based on the textual collection provided in Table 1 is shown in Figure 3 (upper part).

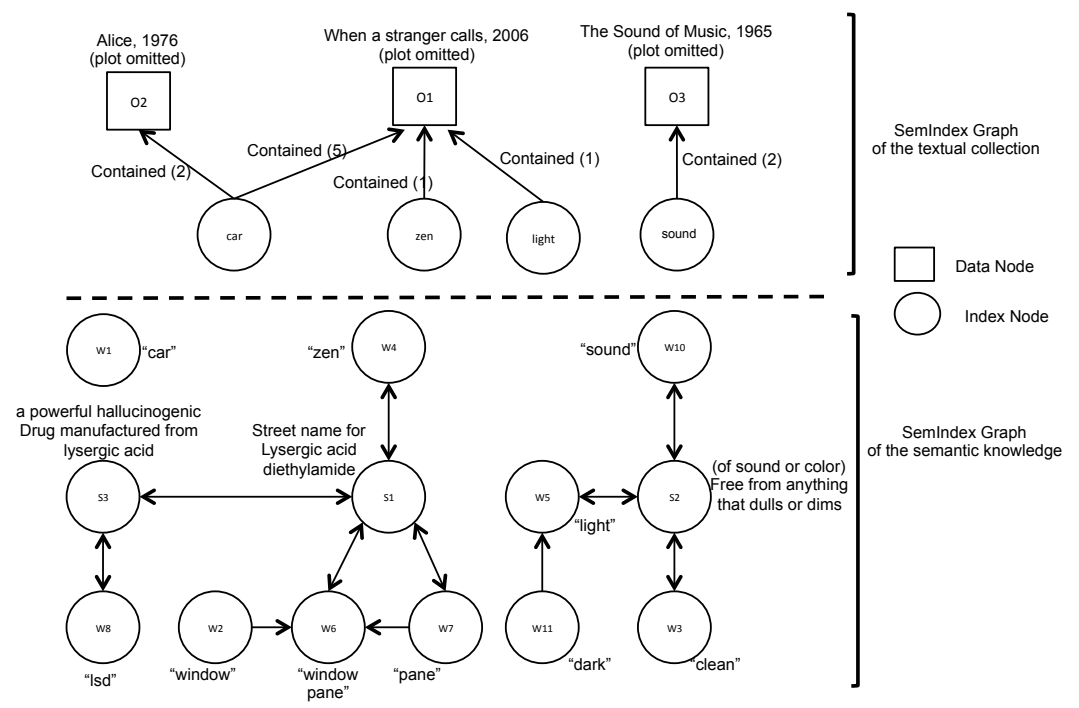

Fig. 3. Part of the SemIndex Graph of the textual collection

Similarly, indexing the semantic knowledge base $G_{k b}$ is also represented as a SemIndex graph $\tilde{G}_{k b}$ that inherits the properties of $G_{k b}$ where:

- $V_{d}$ is an empty set

- $V_{i}$ is a set of vertices/nodes, denoting entities in the given knowledge base and all other searchable terms. For WordNet, $V_{i}$ includes synsets and words as well as other terms that appear in the string value in $G_{k b}$. Thus, $V_{i}$ is a superset of vertices in the knowledge graph $G_{k b}$

- $L$ is a set of edge labels, including those inherited from $G_{k b}$ (e.g., hyponymy, meronymy, etc.), and a special label meronymy* indicating the containment relation from a searchable term to an entity in $V_{i}$

- $S_{e}$ assigns, in addition to previous edges of $G_{k b}$, the meronymy* label from a searchable term to an entity in $V_{i}$

- $W$ is the weighting function assigning a weight (default weight is 1 ) to all nodes and edges.

We assume that connections between searchable terms and entities in $V_{i}$ can be found by the same Natural Language Processing techniques used when indexing the textual collection. For example, in Figure 3 (lower part), we find that word "window" $\left(W_{2}\right)$ is contained in the word "window pane" $\left(W_{6}\right)$. Thus, 
an extra edge labeled meronymy* from $W_{2}$ to $W_{6}$ is inserted into the graph as shown in Figure 3. Note that the NLP algorithms run only on synsets and multi-term words, in order to prevent duplicated nodes to be produced.

2- Coupling Resources: When coupling both indexes, we get one SemIndex graph called $\tilde{G}_{\text {SemIndex }}$. In $\tilde{G}_{\text {SemIndex }}$, only searchable terms have (mainly string) values (which will be defined in Step 3 in the construction procedure). Weights are assigned to all edges and data nodes in the graph. To summarize, $\tilde{G}_{\text {SemIndex }}$ contains:

- $V_{i}$ is a set of index nodes of $\tilde{G}_{A} \cup \tilde{G}_{k b}$

- $V_{d}$ is the set of data nodes of $\tilde{G}_{A}$

- $L$ is a set of labels in the $\tilde{G}_{k b}$ and a special label contained, indicating the containment relationship from a searchable term in $V_{i}$ to an entity in $V_{d}$

- $E$ is a set of ordered pairs of nodes in $V_{i}$ (index edges) and in $V_{d}$ (data edges)

$-S_{v}$ is the function of string values defined only on searchable terms, which is a subset of $V_{i}$

- $S_{e}$ assigns $\tilde{G}_{k b}$ relationship labels to index edges and contained label to index/data edges

- $W$ is the weighting function defined on all nodes in $V_{i} \bigcup V_{d}$ and edges in $E$.

The pseudo-code of constructing $\tilde{G}_{\text {Sem Index }}$ is composed of 7 steps as shown in Algorithm 1. Each step is detailed as follows.

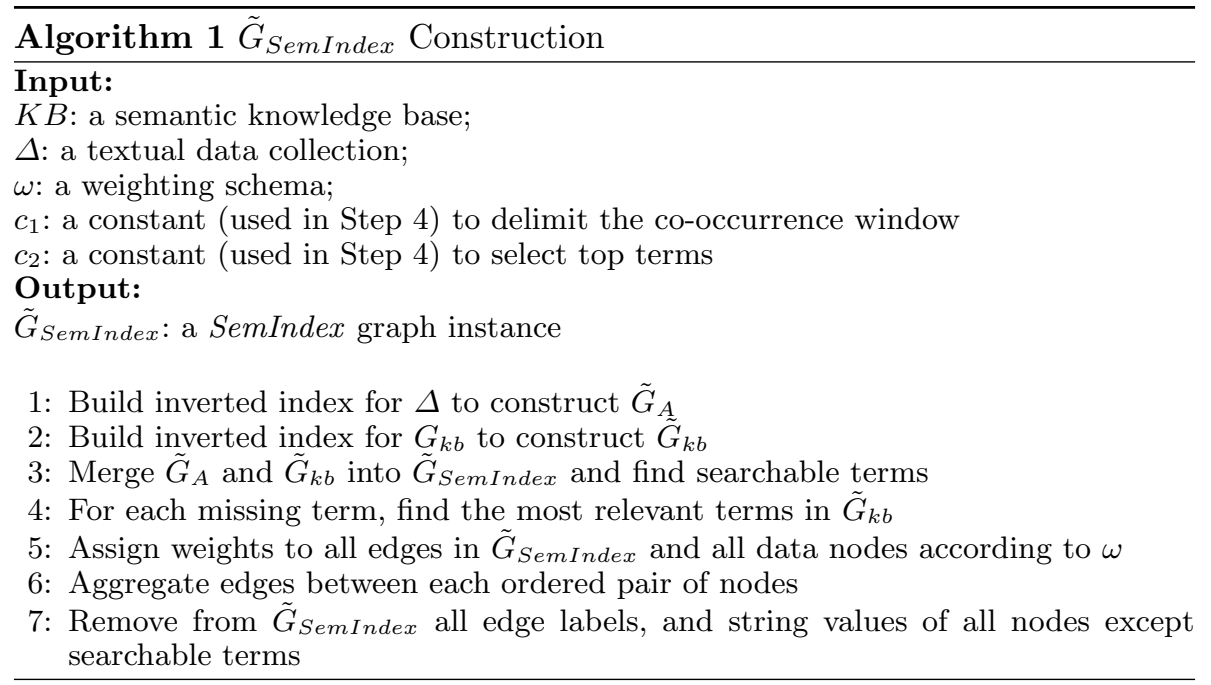

- Step 1: It builds the multi-attributed inverted index on the contents of the textual data collection as a graph $\tilde{G}_{A}$ as defined and illustrated previously.

- Step 2: Given a semantic knowledge graph $G_{k b}$ representing the semantic knowledge base $K B$ given as input, it builds an inverted index for string values of each knowledge base entity, and construct the graph $\tilde{G}_{k b}$.

- Step 3: it combines the two SemIndex graphs. Data nodes in the result graph $\tilde{G}_{\text {SemIndex }}$ are the set $V_{d}$ in $\tilde{G}_{A}$ (denoted as $V_{d}^{A}$ ), while all other nodes are index nodes. This step denotes the searchable terms of $\tilde{G}_{k b}$ as $V_{i}^{k b}$ (which are vertices with one or more outgoing edges labeled contained) and then merges the two sets of searchable terms $V_{i}^{k b}$ and $V_{i}^{A}$ (representing 
the index nodes of $\tilde{G}_{A}$ ) as follows: if string values of two vertices are equal, remove one of them and merge all the connected edges. We use $V_{i}^{+}$to denote the conjunctive set $V_{i}^{k b} \cup V_{i}^{A}$, which is the set of all searchable terms in SemIndex. Figure 4 shows the result of combining the two SemIndex graphs of the sample textual collection and par of the semantic knowledge base of WordNet provided here.

- Step 4: For the missing term problem, we create links from each missing term to one or more closely related terms, with a new edge label refers-to, using a distributional thesaurus ${ }^{8}$ based on the textual collection to mine relativeness between missing terms and used index. We cover the missing term problem in more detail in a dedicated paper.

- Step 5: It assigns weights to edges and textual objects, according to $\omega$. The weight will be used to rank query results. Different weighting schemes can be adopted in our approach, with or without user profiles. We propose below the principles of a simple weighting schema for computing (containment and structural) edge and node weights:

- Containment edges: For a (data) edge from a term to a textual object, its weight is an IR score, such as term frequency. If the textual collection is formatted, this IR score could also be assigned to reflect the importance of a term, e.g., in large font size, in capitalized form, etc. When the textual collection is structured, higher weights are given to terms which appear in important places, e.g., title, author's name, etc.

- Structural edges: The weight of a structural (index) edge is determined by edge label and by the number of edges with the same label from its starting node [21]. Please note that if the knowledge base is hierarchical (which is not the case for WordNet), the level of the edge in the hierarchy can also be taken into consideration [19].

- Nodes: Assign "object rank" to all object nodes, based on metadata of objects, including text length, importance or reliability of data source, its publishing date, query logs, and so on. A PageRank-style weighting schema could also be adapted for Web documents or inter-connected textual objects.

- Step 6: If an ordered pair of vertices is connected with two or more edges, it merges the edges and aggregates the weights. This means that $\tilde{G}_{\text {SemIndex }}$ becomes a graph rather than a multi-graph, which simplifies processing.

- Step 7: It removes edge labels and string values of all nodes except $V_{i}^{+}$ (searchable terms), since they are not required for processing semantic queries, which helps improve SemIdex's scalability.

Figure 4 illustrates an instance of $\tilde{G}_{\text {SemIndex }}$ (without edge and node weights) which is based on the knowledge graph depicted in Figure 2.

\section{Executing queries with SemIndex}

In this section, we define our query model and present a processing algorithm to perform semantic-aware search with the help of SemIndex.

\section{$3.1 \quad$ Queries}

The semantic-aware queries considered in our approach are conjunctive projectionselection queries over $\Delta$ of the form $\pi_{X} \sigma_{P} \ell(\Delta)$ where $X$ is a non empty subset

\footnotetext{
${ }^{8}$ A distributional thesaurus is a thesaurus generated automatically from a corpus by finding words that occur in similar contexts to each other $[11,24]$.
} 


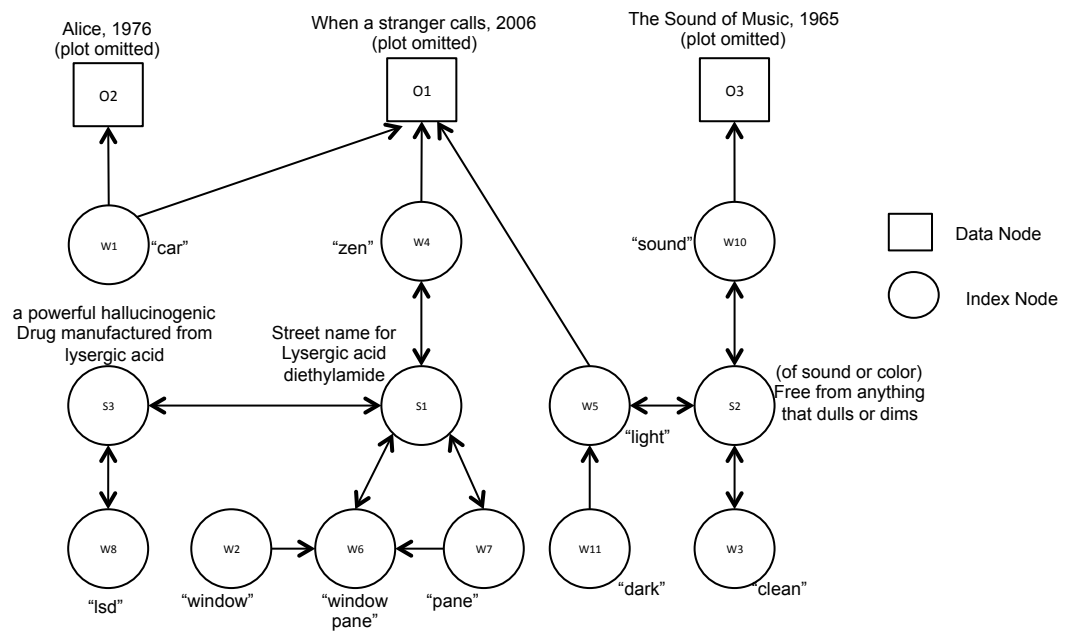

Fig. 4. SemIndex Graph after combining the textual collection and semantic knowledge

of $\mathcal{A}, \ell \in \mathbb{N}$ is a query-type threshold, and $P$ is a conjunctive selection predicate defined as follows.

Definition 4 A selection projection predicate $P$ is an expression, defined on a string-based attribute $A$ in $\mathcal{A}$, of the following forms: $(A \theta a)$, where $a$ is a user-given value (e.g., keyword), and $\theta \in\{=$, like $\}$ whose evaluation against values in $\operatorname{dom}(A)$ is defined. A conjunctive selection projection query is made of a conjunction of selection projection predicates.

According to the value of $\ell$, we consider the four following semantic-aware query types:

- Standard Query: When $\ell=1$, the query is a standard containment query and no semantic information is involved.

- Lexical Query: When $\ell=2$, besides from the previous case, lexical connections, i.e., links between terms, may be involved in the result.

- Synonym-based Query: When $\ell=3$, synsets are also involved in the query processing. Note that there is no direct edge between textual object and synset node.

- Extended Semantic Query: When $\ell \geqslant 4$, the data graph of SemIndex can be explored in all possible ways. When $\ell$ grows larger, the data graph is explored further to reach even more results.

\subsection{Query Answer}

The answer to $q$ in $\Delta$, denoted as $q(\Delta)$, is defined as follows.

Definition 5 Given a SemIndex graph $\tilde{G}$, the query answer $q(\Delta)$ is the set of distinct root nodes of all answer trees. We define an answer tree as a connected graph $T$ satisfying the following conditions:

- (tree structure) $T$ is a subgraph of $\tilde{G}$. For each node in $T$, there exists exactly one directed path from the node to the root object. 
- (root object) The tree root is a data node, and it is the only data node in $T$, which corresponds to the textual object returned to the user.

- (conjunctive selection) For each query term in $S$, its corresponding index node is in the answer tree.

- (height boundary) Height of the tree, i.e., the maximal number of edges between root and each leaf, is no greater than the threshold $\ell$.

- (minimal tree) No node can be removed from $T$ without violating some of the above conditions.

It can be proven that all leaves in the answer tree are query terms, and the number of leaves in $T$ is smaller or equal to $k$, where $k$ is the number of query terms. Also, the maximal in-degree of all nodes in $T$ is at most $k$.

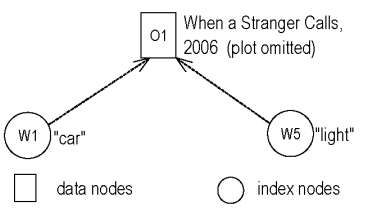

A: Answer Tree of a Standard Query $(\ell=1)$

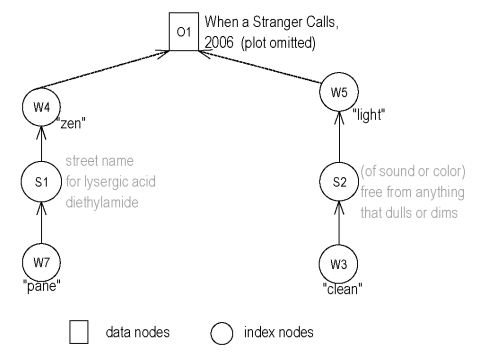

C: Answer Tree of a Synonym-base Query $(\ell=3)$

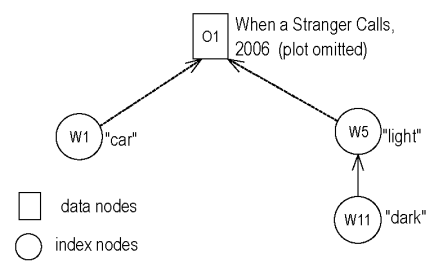

B: Answer Tree of a Lexical Query $(\ell=2)$

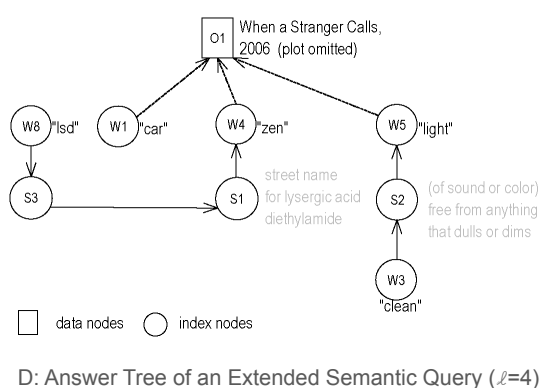

Fig. 5. Answer Trees

According to the value of $\ell$ which serves as an interval radius in theSemIndex graph, various answer trees can be generated for a number of query types, as follows:

- Standard Query: When $\ell=1$, the root of the answer tree is linked directly to all leaves, representing the fact that the result data object contains all query selection terms directly. An example answer tree is shown in Figure 5-A for the query $q: \pi_{\mathcal{A}} \sigma_{A \in \text { ("car", "light") }} \ell_{=1}(\Delta)$.

- Lexical Query: When $\ell=2$, the answer tree contains also lexical connections between selection terms. Figure 5-B is an example answer tree of $q$ : $\pi_{\mathcal{A}} \sigma_{A \in(\text { "car" " } d a r k ")} \ell_{=2}(\Delta)$.

- Synonym-based Query: When $\ell=3$, the answer tree contains, in addition to the two previous cases, the synsets. Note that due to the "minimal tree" restriction, a synset cannot be a leaf node of an answer tree. Thus, if an answer tree contains a synset, the height of the tree is no less than 3 . An example answer tree is shown in Figure 5 -C for $q: \pi_{\mathcal{A}} \sigma_{A \in(\text { "pane", "clean") }} \ell=3(\Delta)$. Synonyms of the two query terms, "zen" and "light", are also contained in the result object $O_{1}$. 
- Extended Semantic Query: When $\ell \geqslant 4$, the answer tree contained additional nodes according to the provided value. An example answer tree

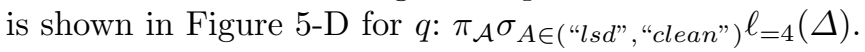

\subsection{Query Processing}

Algorithm 2 is the procedure to process semantic-aware queries, given a set of query terms, terms, and a query-type threshold $\ell$. Function expandNode $(\mathrm{n}, \ell)$ performs the expansion of a node $n$. Basically, it explores the SemIndex graph with Dijkstra's algorithm from multiple starting points (multiple query terms). For each visited node $n$, we store its shortest distances from all starting points (query terms). The path score of a node $n$ to a query term $t$ is the sum of all weights on index edges along the path between $t$ and $n$, thus the shortest distances of $n$ are also the minimal path scores of $n$ to all query terms.

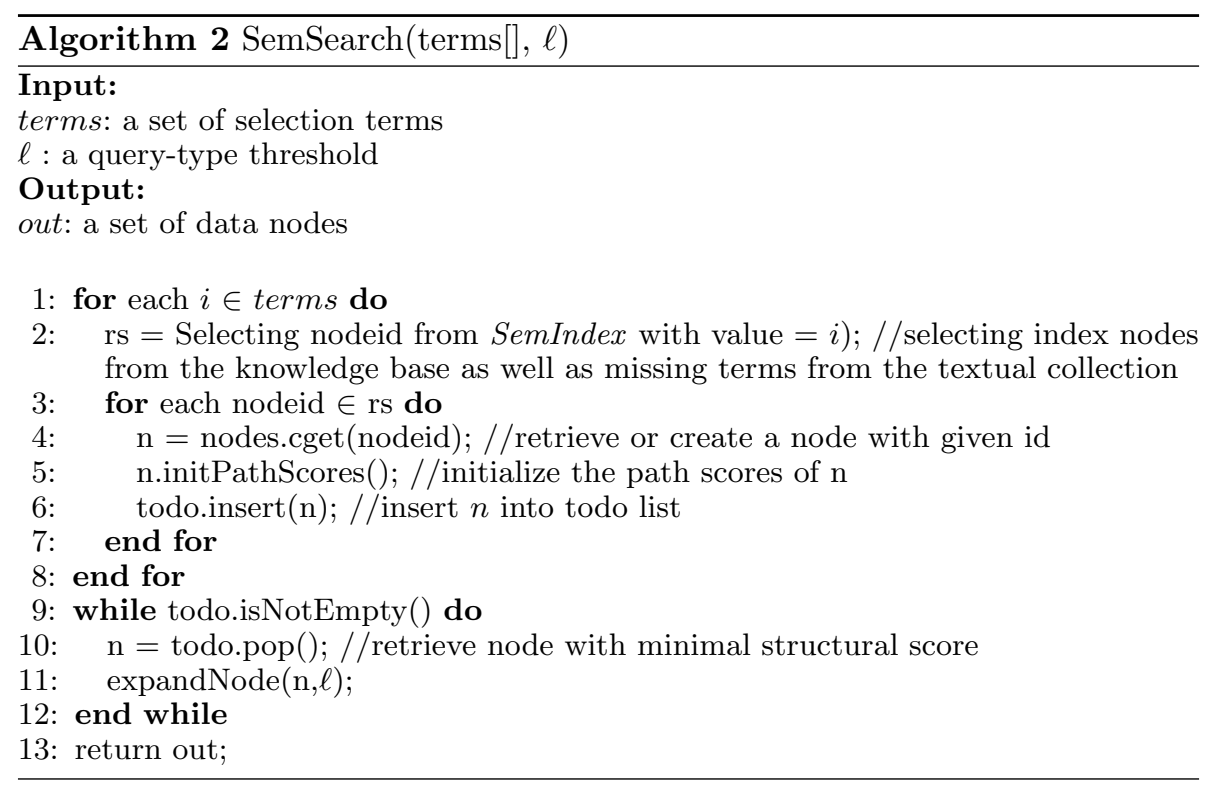

For example in Figure 5-C, the query terms are "pane" and "clean", and the algorithm starts to expand from two nodes $W_{7}$ and $W_{3}$. Path scores of $W_{7}$ are initialized to be a vector $\langle 0, \infty\rangle$, since the shortest distance from $W_{7}$ to "pane" is 0 , but the node is not reachable from "clean". Similarly, the path scores of $W_{3}$ are initially $\langle\infty, 0\rangle$. The minimal path scores must be updated when an edge is explored in the graph. For example, before finding the tree in Figure 5-B, the path scores of node $O_{1}$ is $\langle 2, \infty\rangle$ (assume all edge weights are equal to 1 ), and the path scores of $W_{5}$ are $\langle\infty, 2\rangle$. After exploring the edge from $W_{5}$ to $O_{1}$, the path scores value of $O_{1}$ becomes $\langle 1,2\rangle$, and $O_{1}$ is reachable from all query terms. The algorithm also keeps a todo list, which contains all nodes to be further expanded. The todo list is ordered on structural scores of the nodes. We define the structural score of a node $n$ to be the maximal path score in the tree rooted on $n$, as shown in the following formula.

$$
\text { sscore }_{n}=\max _{t \in \text { rterms }} \text { pathscore }_{n}(t)
$$

where rterms is the set of reachable query terms of $n$. It can be proven that if $n$ is reachable from every term and all path scores of $n$ are minimal, the structural 
score of $n$ must be minimal among all trees rooted on $n$. For each result textual object, the algorithm always returns the answer tree with minimal structural score, thus it is not necessary to prune duplicated query results.

\section{Experiments}

We conducted a set of preliminary experiments to observe the behavior of $S e$ mIndex in weighting, scoring, and retrieving results. In this paper, we only present results related to processing time. We are currently working on experimentally comparing our approach with existing methods.

\subsection{Experimental Setup}

We ran our experiments on a PC with Intel $2 \mathrm{GHz}$ Dual CPU and 2GB memory. SemIndex was physically implemented in a MySQL 5.1 database with the query processor written in Java. We downloaded 90,091 movie plots from the IMDB database $^{9}$, and used WordNet 3.0 as our semantic knowledge base. We build SemIndex on plot contents and movie titles, which means that each textual object is a movie title combined with its plot (cf. Table 2).

Table 2. SemIndex Database Size

\begin{tabular}{|l|c|c|c|}
\hline Database Name & Table Name & Table Size & Table Cardinality (\#Row) \\
\hline \hline IMDB & Data IMDB & $56 \mathrm{M}$ & $90 \mathrm{~K}$ \\
\hline \hline \multirow{5}{*}{ WordNet } & Data Adjective & $3,2 \mathrm{M}$ & $18 \mathrm{~K}$ \\
& Data Verb & $2,8 \mathrm{M}$ & $13 \mathrm{~K}$ \\
& Data Noun & $15,3 \mathrm{M}$ & $82 \mathrm{~K}$ \\
& Data Adverb & $0,5 \mathrm{~K}$ & $3 \mathrm{~K}$ \\
& Index Verb & $0,5 \mathrm{M}$ & $11 \mathrm{k}$ \\
& Index Adverb & $0,2 \mathrm{M}$ & $3,6 \mathrm{~K}$ \\
& Index Noun & $4,8 \mathrm{M}$ & $117 \mathrm{~K}$ \\
& Index Adjective & $0,8 \mathrm{M}$ & $21 \mathrm{k}$ \\
& Index Sense & $7,3 \mathrm{M}$ & $207 \mathrm{~K}$ \\
\hline \hline \multirow{3}{*}{ SemIndex } & Lexicon & $5.8 \mathrm{M}$ & $146 \mathrm{~K}$ \\
& Neighbors & $116 \mathrm{M}$ & $230 \mathrm{~K}$ \\
& PostingList & $340 \mathrm{M}$ & $740 \mathrm{~K}$ \\
\hline
\end{tabular}

\subsection{Query Processing}

In order to test the performance of SemIndex, we manually picked two groups of queries, shown in Table 3 . In the first query group $Q 1$, from $Q 1-1$ to $Q 1-8$, the height of the answer trees is bounded, the number of returned results is limited to 10, and each query contains from 2 to 5 selection terms. In the second group $Q 2$ group, from $Q 2-1$ to $Q 2-4$, queries share the same query terms with different levels of tree height boundary and with an unlimited number of query results. All queries were processed 5 times, retaining average processing time. Detailed statistics are shown in Table 4.

Figure 6 shows query processing time to retrieve the 10000 results of $Q 2-4$. Initial response time is the processing time to output the first result. We break the latter into $\mathrm{CPU}$ and $\mathrm{I} / \mathrm{O}$ time in order to better evaluate the processing costs of the algorithm. Minimal height is the height of the first returned answer tree while the maximal one is the height of the last answer tree allowing to reach the number of expected results. $k, N_{E}$ and $N_{O}$ are respectively the number of query terms, visited entity nodes, and object nodes during query processing.

\footnotetext{
${ }^{9}$ http://imdb.com
} 
Table 3. Sample Queries

\begin{tabular}{|c|c|c|c|}
\hline $\begin{array}{c}\text { Query } \\
\text { Id }\end{array}$ & $\begin{array}{c}\text { Height } \\
\text { Boundary }\end{array}$ & $\begin{array}{c}\text { Max. N\# } \\
\text { of Results }\end{array}$ & $\begin{array}{c}\text { Selection } \\
\text { Terms }\end{array}$ \\
\hline Q1-1 & 4 & 10 & car window \\
Q1-2 & 4 & 10 & reason father \\
Q1-3 & 4 & 10 & car window clean \\
Q1-4 & 4 & 10 & apple pure creative \\
Q1-5 & 4 & 10 & car window clean music \\
Q1-6 & 4 & 10 & death piano radio war \\
Q1-7 & 4 & 10 & car window clean music Tom \\
Q1-8 & 4 & 10 & sound singer stop wait water \\
\hline Q2-1 & 1 & unlimited & car window clean \\
Q2-2 & 2 & unlimited & car window clean \\
Q2-3 & 3 & unlimited & car window clean \\
Q2-4 & 4 & unlimited & car window clean \\
\hline
\end{tabular}

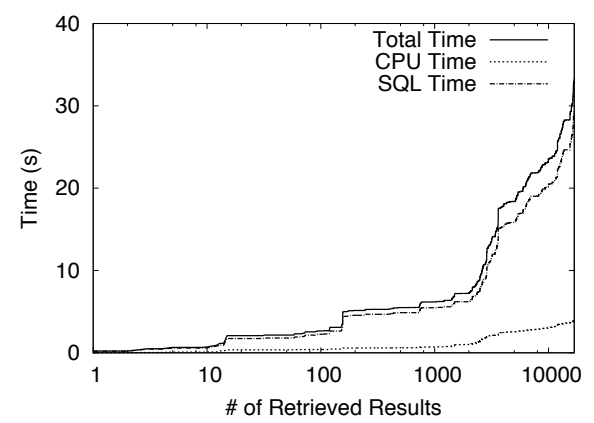

Fig. 6. Processing Time (Q2-4)

From Table 4, we see that most queries are processed within 2 seconds, which is positively encouraging, except for Q2-4, since it is of an extended semantic type, which retrieves 16830 results in about a half minute. In fact, while the total number of textual objects in our plot dataset is around $90 \mathrm{~K}$, Q2-4 visits about $80 \mathrm{~K}$ of object nodes which explains the significant increase in time. Also, we realize that the initial response times of Q1-5 and Q1-6 are quite different. Both of them contain 4 terms and all statistics of the two queries are similar except the minimal tree height. We also observe that for the second query group, the CPU time is dominated by the SQL time, while for the first query group, CPU cost is dominant. We analyzed the difference and found that, for the second query group, the algorithm cannot stop until all visited entity nodes are queried and the whole search space is examined. However for the first query group, since the number of query results is limited, the algorithm stops whenever it has found enough answer trees. Thus, for the first group of queries, although large $N_{E}$ and $N_{O}$ suggest large CPU time to create those nodes in memory, yet most of the nodes are not revisited for expansion before the algorithm stops, which significantly reduces overall query processing time.

Table 4. Processing Statistics

\begin{tabular}{|c|c|c|c|c|c|c|c|c|c|c|}
\hline Queryid & \begin{tabular}{|c|} 
Initial \\
Response \\
Time (ms)
\end{tabular} & \begin{tabular}{|c|} 
Total \\
Process \\
Time $(\mathrm{ms})$
\end{tabular} & \begin{tabular}{|c|} 
Min. \\
Height
\end{tabular} & \begin{tabular}{|c|} 
Max. \\
Height
\end{tabular} & \begin{tabular}{|c|}
$\#$ of \\
Results \\
Returned
\end{tabular} & $\begin{array}{c}\text { SQL } \\
\text { Time (ms) }\end{array}$ & \begin{tabular}{|c|} 
CPU \\
Time (ms)
\end{tabular} & $\mathbf{k}$ & $N_{E}$ & $N_{O}$ \\
\hline Q1-1 & \begin{tabular}{|l|}
26 \\
\end{tabular} & 27 & 1 & 1 & 10 & 15 & 12 & 2 & 399 & 3346 \\
\hline Q1-2 & 39 & 40 & 1 & 1 & 10 & 21 & 19 & 2 & 252 & 9386 \\
\hline Q1-3 & 25 & 148 & 1 & 3 & 10 & 87 & 60 & 3 & 2528 & 7646 \\
\hline Q1-4 & 90 & 315 & 4 & 4 & 10 & 236 & 79 & 3 & 2812 & 11543 \\
\hline Q1-5 & 694 & 700 & 4 & 4 & 10 & 224 & 475 & 4 & 7849 & 32776 \\
\hline Q1-6 & 175 & 511 & 3 & 4 & 10 & 193 & 318 & 4 & 5071 & 38988 \\
\hline Q1-7 & 1223 & 1371 & 4 & 4 & 10 & 643 & 728 & 5 & 9505 & 50747 \\
\hline Q1-8 & 1469 & 1555 & 4 & 4 & 10 & 467 & 1076 & 5 & 12449 & 45715 \\
\hline Q2-1 & 18 & 18 & 1 & 1 & 2 & 11 & 7 & 3 & 3 & 3803 \\
\hline Q2-2 & 25 & 208 & 1 & 2 & 6 & 191 & 16 & 3 & 533 & 4394 \\
\hline Q2-3 & 21 & 842 & 1 & 3 & 515 & 678 & 164 & 3 & 1564 & 34564 \\
\hline Q2-4 & 200 & 31991 & 1 & 4 & 16830 & 27793 & 4198 & 3 & 19007 & 79997 \\
\hline
\end{tabular}




\section{Related Work}

Including semantic processing in inverted indexes to enhance data search capabilities has been investigated in different approaches: i) including semantic knowledge into an inverted index, ii) including full-text information into the semantic knowledge base, and iii) building an integrated hybrid structure.

The first approach consists in adding additional entries in the index structure to designate semantic information. Here, the authors in [12] suggest extending the traditional (term, docIDs) inverted index toward a (term, context, docIDs) structure where contexts designate synsets extracted from WordNet, associated to each term in the index taking into account the statistical occurrences of concepts in Web document [1]. An approach in [26] extends the inverted index structure by adding additional pointers linking each entry of the index to semantically related terms, (term, docIDs, relatedTerms). Yet, the authors in $[12,26]$ do not provide the details on how concepts are selected from WordNet and how they are associated to each term in the index.

Another approach to semantic indexing is to add words as entities in the ontology $[1,18,22]$. For instance, adding triples of the form word occurs-incontext concept, such that each word can be related to a certain ontological concept, when used in a certain context. Following such an approach: i) the number of triples would naturally explode, given that ii) query processing would require reaching over the entire left and the right hand sides of this occurs-incontext index, which would be more time consuming [1] than reading on indexed entry such as with the inverted index.

A third approach to semantic indexing consists in building an integrated hybrid structure: combining the powerful functionalities of inverted indexing with semantic processing capabilities. To our knowledge, one existing method in [1] has investigated this approach, introducing a joint index over ontologies and text. The authors consider two input lists: containing text postings (for words or occurrences), and lists containing data from ontological relations (for concept relations). The authors tailor their method toward incremental query construction with context-sensitive suggestions. They introduce the notion of context lists instead of usual inverted lists, where a prefix contains one index item per occurrence of a word starting with that prefix, adding an entry item for each occurrence of an ontological concept in the same context as one of these words, producing an integrated 4-tuples index structure (prefix, terms) $\leftrightarrow$ (term, context, concepts). The method in [1] is arguably the most related to our study, with one major difference: the authors in [1] target semantic fulltext search and indexing with special emphasis on IR-style incremental query construction, whereas we target semantic search in textual databases: building a hybrid semantic inverted index to process DB-style queries in a textual DB.

\section{Conclusions and Future Work}

In this paper, we introduce a new semantic indexing approach called SemIndex, creating a hybrid structure using a tight coupling between two resources: a general purpose semantic network, and a standard inverted index defined on a collection of textual data, represented as (multi)graphs. We also provide an extended query model and related processing algorithms, using SemIndex to allow semantic-aware querying. Preliminary experimental results are promising, demonstrating the scalability of the approach in querying a large textual data collection (IMBD) coined with a full-fledge semantic knowledge base (WordNet). We are currently completing an extensive experimental study to evaluate SemIndex's properties in terms of: i) genericity: to support different types of 
textual (structured, semi-structured, NoSQL) databases, ranking schema, and knowledge-bases, ii) effectiveness: evaluating the interestingness of semanticaware answers from the user's perspective, and iii) efficiency: to reduce index's building and updating costs as well as query processing cost. The systems physical structure (in addition to the logical designs provided in this paper) will also be detailed in an upcoming study.

\section{Acknowledgements}

This study is partly funded by: Bourgogne Region program, CNRS, and STIC AmSud project Geo-Climate XMine, and LAU grant SOERC-1314T012.

\section{References}

1. H. Bast and B. Buchhold, An index for efficient semantic full-text search, 22nd ACM Int. Conf. on CIKM, 2013, pp. 369-378.

2. A. Burton-Jones and et al., A heuristic-based methodology for semantic augmentation of user queries on the web, Conceptual Modeling - ER 2003, LNCS, vol. 2813, 2003, pp. 476-489.

3. C. Carpineto and et al., Improving retrieval feedback with multiple term-ranking function combination, ACM Trans. Inf. Syst. 20 (2002), no. 3, 259-290.

4. K. Chandramouli and et al., Query refinement and user relevance feedback for contextualized image retrieval, 5th International Conference on Visual Information Engineering, 2008, pp. 453-458.

5. P. Cimiano and et al., Towards the self-annotating web, 13th Int. Conf. on WWW, 2004, pp. 462-471.

6. S. Das and et al., Making unstructured data sparql using semantic indexing in oracle database, IEEE 29th ICDE (2012), 1405-1416.

7. Erika F. de Lima and Jan O. Pedersen, Phrase recognition and expansion for short, precision-biased queries based on a query log, 22nd int. Conf. ACM SIGIR, 1999, pp. 145-152.

8. C. Fellbaum, Wordnet an electronic lexical database, MIT Press, May 1998.

9. D. Florescu and et al., Integrating keyword search into xml query processing, Comput. Netw. 33 (2000), no. 1-6, 119-135.

10. W. B. Frakes and R. A. Baeza-Yates (eds.), Information retrieval: Data structures and algorithms, Prentice-Hall, 1992.

11. G. Grefenstette, Explorations in automatic thesaurus discovery, Kluwer Pub., 1994.

12. S. Kumar and et al., Ontology based semantic indexing approach for information retrieval system, Int. J. of Comp. App. 49 (2012), no. 12, 14-18.

13. Y. Li and et al., Term disambiguation in natural language query for xml, 7 th Int. Conf. on FQAS, 2006, pp. 133-146.

14. C. Mishra and N. Koudas, Interactive query refinement, 12th Int. Conf. on EDBT, 2009, pp. 862-873.

15. R. Navigli, Word sense disambiguation: A survey, ACM Comput. Surv. 41 (2009), no. 2, 10:1-10:69.

16. R. Navigli and G. Crisafulli, Inducing word senses to improve web search result clustering, Int. Conf. on Empirical Methods in Natural Language Processing, 2010, pp. 116-126.

17. S. Nguyen and et al., Semantic evaluation of search result clustering methods, Intelligent Tools for Building a Scientific Information Platform, Studies in Computational Intelligence, vol. 467, 2013, pp. 393-414.

18. R. Navigli Paola and et al., Extending and enriching wordnet with ontolearn, Int. Conf. on GWC 2004, 2004, pp. 279-284.

19. P. Resnik, Using information content to evaluate semantic similarity in a taxonomy, 14th Int. Conf. on Artificial intelligence, 1995, pp. 448-453.

20. G. Salton and C. Buckley, Improving retrieval performance by relevance feedback, Readings in Information Retrieval, 1997, pp. 355-364.

21. M. Sussna, Word sense disambiguation for free-text indexing using a massive semantic network, 2nd int. ACM Conf. on CIKM, 1993, pp. 67-74.

22. P. Velardi and et al., Ontolearn reloaded: A graph-based algorithm for taxonomy induction, Computational Linguistics 39 (2013), no. 3, 665-707. 
23. E. M. Voorhees, Query expansion using lexical-semantic relations, 17th int. ACM conf. on SIGIR, 1994, pp. 61-69.

24. J. Weeds and et al., Characterising measures of lexical distributional similarity, 20th int. conf.on Computational Linguistics, 2004.

25. H. Wen and et al., Clustering web search results using semantic information, 2009 Int. Conf. on Machine Learning and Cybernetics, vol. 3, 2009, pp. 1504-1509.

26. S. Zhong and et al., A design of the inverted index based on web document comprehending, JCP 6 (2011), no. 4, 664-670. 